\title{
Phase transitions in an anyon gas
}

\section{R. B. MacKenzie ${ }^{2}$, F. Nebia-Rahal ${ }^{3}$, Manu Paranjape $^{1 *}{ }^{\dagger}$}

Groupe de physique des particules

Département de physique

Université de Montréal

C. P. 6128, succ. centre-ville

Montréal, Québec, Canada, H3T 1L7

E-mail: ${ }^{1}$ paranjelps.umontreal.ca

E-mail: ${ }^{2}$ rbmackelps . umontreal.ca

E-mail: ${ }^{3}$ faizanralps.umontreal.ca

\section{J. Richer}

Réseau québecois de calcul de haute performance

DGTIC, Université de Montréal

C.P. 6128, Succ. Centre-ville, Montréal, Québec, Canada, H3C 3J7

E-mail: jacques.richer@umontreal.ca

We report on our numerical, Monte Carlo simulation study of a gas of closed loops on a 3 dimensional lattice in the presence of a topological term which computes the linking number of the virtual loops. The topological term converts the particles in the loop into anyons. The term is equivalent to the Chern-Simons term for the corresponding abelian-Higgs model, in a strong coupling limit. The system exhibits a phase transition as a function of the anyon mass, as this mass becomes small, the vacuum is saturated by a so-called infinite length anyon loop configuration. We find that the Chern-Simons term has no effect on the Wilson loop however, it contributes to the 't Hooft loop by exactly it's linking number with all of the dynamical anyon loops. We find the interesting result that both the Wilson loop and the 't Hooft loop both exhibit a perimeter law behaviour even though there are no massless particles in the theory.

The 30th International Symposium on Lattice Field Theory

June $24^{\wedge} 29,2012$

Cairns, Australia

\footnotetext{
${ }^{*}$ Speaker.

${ }^{\dagger}$ We thank NSERC of Canada for partial financial support.
} 


\section{INTRODUCTION}

We report on our study a 3-dimensional lattice loop gas [1,2], with a topological interaction which counts the total linking number of the loops with each other. The only interactions they feel are a statistical, Aharonov-Bohm [3] type interaction and an infinite short range repulsion between particles (and antiparticles) which prohibits the particles to come close to each other. The Aharonov-Bohm interaction causes a change of phase of the wave function when one particle encircles another, giving a phase dependent on the coefficient of the topological term. The topological term contributes a phase $e^{i \kappa}$ to the corresponding amplitude, where $\kappa$ is the coefficient of the topological term in the action.

In [1], it was proposed that the lattice loop gas is an effective theory describing the asymptotic strong-coupling limit of the Abelian Higgs model. This model contains fundamental scalar and gauge particles and also vortices. In the asymptotic strong-coupling limit the fundamental particles become infinitely massive: they decouple from the theory, leaving only vortices. The topological interaction between lattice loops corresponds in the Abelian Higgs model to the addition of a Chern-Simons term.

The Euclidean action is no longer real, the Chern-Simons term is an imaginary term in the action. This is an impediment to numerical simulation of the theory using the Monte Carlo. However, we can use the real part of the action to give us the probability distribution. Then the Chern-Simons term is simply a bounded unimodular phase which can be integrated against the measure that is defined by the real part of the action. It does give rise to the familiar sign problem.

The Chern-Simons term for the configurations that we are left with (non-intersecting closed loops on the lattice) is simply equal to twice the total linking number of all the loops [4]. We can see this quite easily by observing that since the Chern-Simons integrand is proportional to the magnetic field, the integral reduces to a set of line integrals along the vortex flux lines:

$$
S_{C S}=\frac{\kappa}{4 \pi^{2}} \int d^{3} x \varepsilon^{\mu \nu \lambda} A_{\mu} \partial_{v} A_{\lambda}=\frac{\kappa}{2}\left(\sum_{C_{i}} N_{L}\left(C_{i}\right)\right)
$$

where $N_{L}\left(C_{i}\right)$ is exactly the linking number of vortex line $C_{i}$ with all of the other vortex lines. Summing over all the curves clearly gives twice the total linking number of all the vortex loops, $\sum_{C_{i}} N_{L}\left(C_{i}\right)=2 N_{T}$, where $N_{T}$ is the total linking number of the configuration of vortex loops; i.e.,

$$
\frac{\kappa}{2}\left(\sum_{C_{i}} N_{L}\left(C_{i}\right)\right)=\kappa N_{T}
$$

\section{Computing the linking number}

The closed non-intersecting loops were generated on a body centered cubic (bcc) lattice of size $100^{3}$ by placing the cube roots of unity randomly on the vertices, with all points on the surface of the lattice assigned the same value. The lattice can be thought of as filling space with (non-regular) tetrahedra. Each cube contains six pyramids; adding the diagonal of the cubic sides in a systematic way throughout the lattice divides each pyramid into two non-regular tetrahedra (see [1] for details). If the change in phase of the cube roots of unity around one of the triangular faces of a tetrahedron 
is equal to $\pm 2 \pi$, we say a length of vortex flux tube has entered or exited the tetrahedron through that face. If the flux entered the tetrahedron it is easy to see that whatever cube root of unity is placed on the fourth vertex of the tetrahedron, the flux must exit the tetrahedron through one of the other faces. But then it enters another tetrahedron since the tetrahedra fill space. The loop must close, since the boundary condition used means no surface triangle has a flux passing through it. The loops so defined exist in the dual lattice to the initial tetrahedral lattice.

To compute the linking number of any given loop with all of the other loops, we need to simply compute the flux that passes through the given loop, since each other loop that links with it carries one unit of flux. The flux that passes through a loop that is defined along the links and vertices of the original lattice is trivially calculated: we simply calculate the change of phase of the cube roots of unity as we pass through the vertices of such a loop. Thus if we can systematically deform the given vortex loop on the dual lattice to a loop on the original lattice, the calculation is straightforward. The calculation of the linking number via the flux that passes through the deformed loop also calculates the linking number of the deformed loop with the original loop, which defines the self-linking number of the original vortex loop. We must subtract this self-linking number from the calculation of the linking number of the deformed loop.

However, the self-linking number is in fact the ordinary linking number of the deformed loop with the original loop. There is a simple way to compute this linking number, using knot theory [5].

We can project the knot onto a two dimensional plane, keeping track only of the the sense of the crossings of the segments of one loop with the other in the projection. Then a sum of the association of \pm 1 to the crossings, depending on which segment of which loop is on top of the other and its direction, gives the linking number. The important point is to choose the direction of projection that will yield the most simplifying two dimensional projection.

Projecting along the diagonal of the lattice (the $(1,1,1)$ direction in coordinate space) actually yields exactly a regular triangular lattice on the projected two plane. The original loop, which passes through the dual lattice, projects to the dual lattice of the two dimensional triangular lattice, while the deformed loop, of course, projects directly to the links of the triangular lattice

Thus the crossings are unambiguous and occur at a small, finite number of intersection points. It is easy to keep track of the segments of each loop, and their relative heights. This simply amounts to a re-indexing of the data which is already stored in the computer, in a new system of coordinates given by the triangular lattice in the two dimensional projected plane and the height along the $(1,1,1)$ direction.

\section{Calculating in the Chern-Simons Theory}

We obtain the set of equilibrium configurations by using the Boltzmann weight given by the total length of the vortex loops. Thus the Boltzmann weight is unaffected by the Chern-Simons term. This is a classic problem for numerical simulations; it arises in theories with fermions where it is called the sign problem, but also in the context of topological terms which are odd under time reversal; a fuller explanation of this is provided, for example, in [6]. The solution in the present case is based on the following logic. The (Euclidean) Feynman path integral corresponds to the 
integral

$$
\langle\mathscr{O}\rangle=\int \mathscr{D} \varphi e^{-S_{E} / \hbar} \mathscr{O}
$$

over the space of field configurations, with appropriate boundary conditions, to calculate a quantum amplitude $\langle\mathscr{O}\rangle$. However, the actual measure on this space is not specified.

The important property that the measure used to construct the quantum field theory must satisfy, is that it respect all the symmetries of the theory that one is trying to construct. We use the full real part of the action, which provides an appropriate measure. We use the approximation for the action, the total length of the vortex loops for the field configurations that we consider. The full real part of the action actually has more symmetry than the theory with the Chern-Simons term added since parity and time reversal are not symmetries of the full theory. However this does not cause any problem since all amplitudes are calculated with the Chern-Simons term inserted.

Hence we use the full real part of the action to define the measure on the space of field configurations, and in the context of the Monte-Carlo method, the set of equilibrium configurations, say a total number $N$. After this, the Chern-Simons term simply gives a uni-modular phase that can be integrated against this measure. When calculating actual matrix elements of an operator, we must calculate the average of the operator with the phase coming from the Chern-Simons term inserted and then divide by the partition function, again defined with the same phase inserted. This procedure is called reweighting. Explicitly we get

$$
\langle\mathscr{O}\rangle \approx\langle\mathscr{O}\rangle_{N}=\frac{\sum_{i=1}^{N} \mathscr{O}\left(\mathscr{C}_{i}\right) e^{i S_{C S}\left(\mathscr{C}_{i}\right)}}{\sum_{i=1}^{N} e^{i S_{C S}\left(\mathscr{C}_{i}\right)}}
$$

where $\mathscr{C}_{i}$ stands for the $i$ th configuration. Normally, both numerator and denominator contain a factor of $1 / N$; however, here it cancels between them.

\subsection{Chern-Simons term}

The Chern-Simons term in the functional integral gives exactly the linking number of all the dynamical loops. We use knot theoretic techniques to compute the average of the Chern-Simons term in the set of equilibrium configurations, for different values of the mass $\mu$. We see that the expectation value drops to zero remarkably quickly as the system passes through the transition at about $\mu=0.152$ for decreasing $\mu$. The graph for the expectation value of the Chern-Simons term is given in Fig. 1 for various values of $\mu$. This average of the Chern-Simons term serves as the partition function when computing the expectation value of any operator. Clearly proceeding to values of $\kappa \gtrsim 0.08$ is not possible for $\mu \lesssim 0.15$.

\subsection{Wilson loop}

The Wilson loop [7] is defined as the expectation value of the operator

$$
W=e^{-i(q / e) \oint A_{\mu} d x^{\mu}}
$$

where $e$ is the fundamental charge in the model and the integral in the exponent goes along a closed, fixed rectangular path of width $L$ and length $T$. For a given configuration $\mathscr{C}_{i}$, the exponent in the 


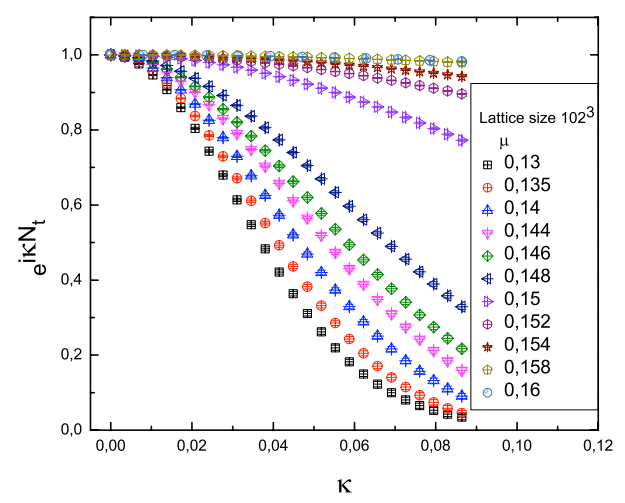

Figure 1: (color online) The average value of the Chern-Simons term as a function of $\kappa$.

Wilson loop is equal to the linking number of the curve defining the Wilson loop and the dynamical vortex loops in the configuration, say $N_{W L}\left(\mathscr{C}_{i}\right)$,

$$
W\left(\mathscr{C}_{i}\right)=e^{-i(2 \pi q / e) N_{W L}\left(\mathscr{C}_{i}\right)}
$$

The calculation of the expectation value of the Wilson loop can be done by first calculating the average value of the Wilson loop for fixed total linking number, and then performing the sum over these average values, weighted by the number of configurations with the fixed total linking number. Using the notation $\mathscr{N}\left(N_{T}\right)$ for the number of configurations with fixed total linking number $N_{T}$, and $\mathscr{C}_{i, N_{T}}$ as an index for these configurations, we have:

$$
\begin{aligned}
\langle W\rangle & =\frac{\sum_{i} W\left(\mathscr{C}_{i}\right) e^{i \kappa N_{T}\left(\mathscr{C}_{i}\right)}}{\sum_{i} e^{i \kappa N_{T}\left(\mathscr{C}_{i}\right)}} \\
& =\frac{\sum_{N_{T}} \mathscr{N}\left(N_{T}\right)\left(\frac{1}{\mathscr{N}\left(N_{T}\right)} \sum_{\mathscr{C}_{i, N_{T}}} W\left(\mathscr{C}_{i, N_{T}}\right)\right) e^{i \kappa N_{T}}}{\sum_{N_{T}} \mathscr{N}\left(N_{T}\right) e^{i \kappa N_{T}}} .
\end{aligned}
$$

The term in parentheses in the numerator is the average value of the Wilson loop with fixed total linking number. If this is independent of the value of the total linking number, then it comes out of the sum, and in fact the sums in the numerator and denominator cancel, yielding

$$
\langle W\rangle=\frac{1}{\mathscr{N}\left(N_{T}\right)} \sum_{\mathscr{C}_{i, N_{T}}} W\left(\mathscr{C}_{i, N_{t}}\right)
$$

which is in fact independent of $\kappa$. This is exactly what we find with our numerical simulation. In Fig. 2 we plot the value of the Wilson loop for different values of $\mu$ as a function of $\kappa$, at a fixed value $2 \pi q / e=0.18 \pi$. Evidently, the average of the Wilson loop does not depend on $\kappa$ for any value of $\mu$ for $\kappa \lesssim 0.08$. There is an apparent dependence in the graphs for small $\mu$, as $\kappa$ exceeds the value $\sim 0.08$. However, at this point, as we can see from Fig. 1 the value of the Chern-Simons becomes very small, and we can no longer trust the numerical results. 


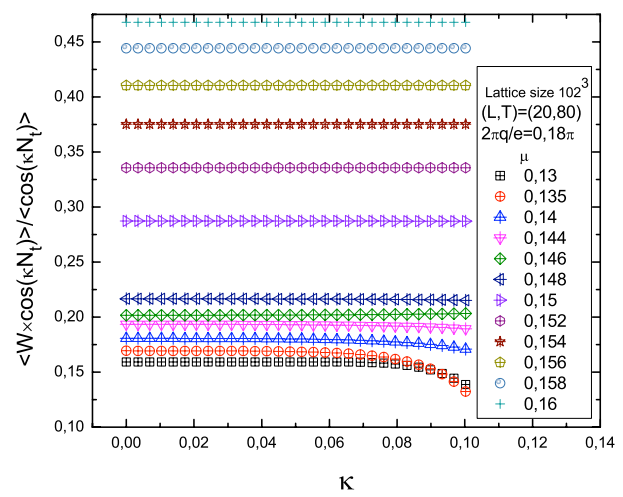

Figure 2: (color online) The Wilson loop for various values of the coefficient of the Chern-Simons term: $\kappa$.

\section{3 't Hooft loop}

The 't Hooft loop [8] corresponds to the insertion of a singular magnetic flux tube along a contour of a fixed rectangular loop of width $L$ and length $T$. The Monte Carlo method of generating the equilibrium configurations is unchanged, using as before only the real part of the full action, with the (infinite) action of the 't Hooft loop subtracted off and with our strong-coupling approximation. Then the equilibrium configurations are comprised of configurations of closed vortex loops appended by the 't Hooft loop. In the presence of the Chern-Simons term, the 't Hooft loop simply adds $\kappa N^{\prime}{ }_{t H L}$ to the action, where $N_{t H L}$ is the linking number of the 't Hooft loop with all the dynamical vortex loops. Hence the 't Hooft loop is given by the average

$$
\left\langle{ }^{\prime} t H\right\rangle=\frac{\sum_{\mathscr{C}_{i}} e^{i \kappa N_{t H}} e^{-S_{E}+i \kappa N_{T}}}{\sum_{\mathscr{C}_{i}} e^{-S_{E}+i \kappa N_{T}}}
$$

In Fig. 3 we plot the average value of the 't Hooft loop as a function of $\kappa$, for various values of the mass $\mu$. The points in the graphs beyond $\kappa=0.08$ should not be trusted since the errors are not under control, as the average value of the Chern-Simons term in the denominator, as we apply Eqn. (3.2), becomes vanishingly small. We note that in contrast with the Wilson loop, the 't Hooft loop has a clear dependence on the coefficient of the Chern-Simons term.

\section{DISCUSSION AND CONCLUSIONS}

The main result that we find is that the Wilson loop [7] is independent of the Chern-Simons term (for sufficiently small values of its coefficient) while the 't Hooft loop [8] is not. In fact for the 't Hooft loop the contribution to the free energy is zero in the absence of the Chern-Simons term but proportional to its coefficient in its presence. The free energy for the Wilson loop is simply independent of the Chern-Simons term.

These results are surprising; but, there is a more interesting result. We have found that both the Wilson loop and the 't Hooft loop have perimeter law behaviour in both phases of the theory. This is remarkable since there is a theorem which states that a perimeter law for both of these order 


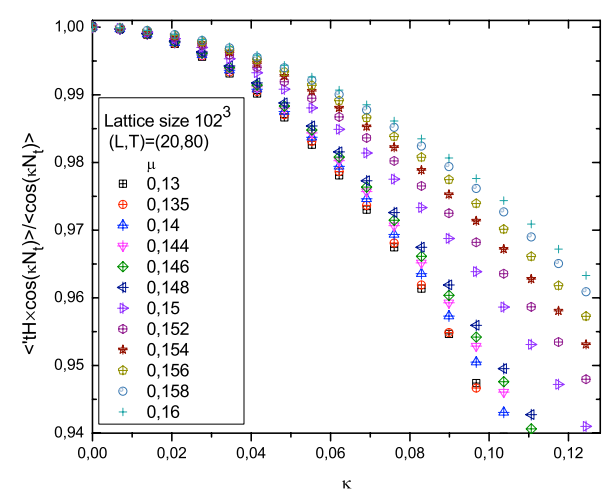

Figure 3: (color online) The average value of the 't Hooft loop in the presence of a Chern-Simons term as a function of $\kappa$.

parameters requires the existence of massless particles [9]; however, we have no massless particles in the theory. The possible explanation for this is that the theorem is circumvented since we have a statistical long range interaction between the anyons.

\section{ACKNOWLEDGEMENTS}

We thank NSERC of Canada for financial support. We also thank the Réseau québécois de calcul de haute performance (RQCHP) for providing us with the computational resources required for this work.

\section{References}

[1] R. MacKenzie, F. Nebia-Rahal, M.B. Paranjape, "Phase transitions in a 3 dimensional lattice loop gas," Phys. Rev. D81, 114505 (2010), [arXiv:0710.3236 [hep-lat]].

[2] R. MacKenzie, F. Nebia-Rahal, M. B. Paranjape and J. Richer, "Phase transitions in a gas of anyons," Phys. Rev. D 82, 074506 (2010) [arXiv:1007.3064 [hep-lat]].

[3] Y. Aharonov and D. Bohm Phys. Rev. 115, 485-491 (1961).

[4] F. Wilczek and A. Zee, Phys.Rev. Lett. 51, 2250 (1983).

[5] R.C. Lacher and D.W. Sumners, "Data structures and algorithms for computation of topological invariants of entanglements: Link, Twist, and Writhe, Computer Simulation of Polymers" (R.J. Roe, ed.), Prentice Hall, Englewood Cliffs, NJ (1990), pp. 365-373.

[6] G. Alexanian, R. MacKenzie, M. B. Paranjape and J. Ruel, "Path integration and perturbation theory with complex Euclidean actions," Phys. Rev. D77, 105014 (2008) [arXiv:0802.0354 [hep-th]].

[7] K. Wilson, Phys. Rev. D10, 2445 (1974).

[8] G. 't Hooft, “On The Phase Transition Towards Permanent Quark Confinement,” Nucl. Phys. B 138, 1 (1978).

[9] A. Ukawa, P. Windey and A. H. Guth, "Dual Variables For Lattice Gauge Theories And The Phase Structure Of Z(N) Systems," Phys. Rev. D21, 1013 (1980). 